\title{
DEA Scores' Confidence Intervals with Past-Present and Past-Present-Future Based Resampling
}

\author{
Kaoru Tone ${ }^{1}$, Jamal Ouenniche ${ }^{2}$ \\ ${ }^{1}$ National Graduate Institute for Policy Studies, Tokyo, Japan \\ ${ }^{2}$ University of Edinburgh, Business School, Edinburgh, UK \\ Email: tone@grips.ac.jp, Jamal.Ouenniche@ed.ac.uk
}

Received 8 January 2016; accepted 5 March 2016; published 8 March 2016

Copyright (C) 2016 by authors and Scientific Research Publishing Inc.

This work is licensed under the Creative Commons Attribution International License (CC BY). http://creativecommons.org/licenses/by/4.0/

(c) (i) Open Access

\begin{abstract}
In data envelopment analysis (DEA), input and output values are subject to change for several reasons. Such variations differ in their input/output items and their decision-making units (DMUs). Hence, DEA efficiency scores need to be examined by considering these factors. In this paper, we propose new resampling models based on these variations for gauging the confidence intervals of DEA scores. The first model utilizes past-present data for estimating data variations imposing chronological order weights which are supplied by Lucas series (a variant of Fibonacci series). The second model deals with future prospects. This model aims at forecasting the future efficiency score and its confidence interval for each DMU. We applied our models to a dataset composed of Japanese municipal hospitals.
\end{abstract}

\section{Keywords}

Data Variation, Resampling, Confidence Interval, Past-Present-Future DEA, Hospital

\section{Introduction}

DEA is a non-parametric methodology for performance evaluation and benchmarking. Since the publication of the seminal paper by Charnes, Cooper and Rhodes [1], DEA has witnessed numerous developments, some of which are motivated by theoretical considerations and others motivated by practical considerations. The focus of this paper is on practical considerations related to data variations. The first practical issue is the lack of a statistical foundation for DEA which was laid down by Banker [2] who proved that DEA models could be viewed as maximum likelihood estimation models under specific conditions and then Banker and Natarajan [3] proved that 
DEA provides a consistent estimator of arbitrary monotone and concave production functions when the (onesided) deviations from such a production function are degraded as stochastic variations in technical inefficiency. Afterwards the treatment of data variations has taken a variety of forms in DEA. In fact, several authors investigated the sensitivity of DEA scores to data variations in inputs and/or outputs using sensitivity analysis and super-efficiency analysis. For example, Charnes and Neralić [4] and Neralić [5] used conventional linear programming-based sensitivity analysis under additive and multiplicative changes in inputs and/or outputs to investigate the conditions under which the efficiency status of an efficient DMU is preserved (i.e., basis remains unchanged), whereas Zhu [6] performed sensitivity analysis using various super-efficiency DEA models in which a test DMU is not included in the reference set. This sensitivity analysis approach simultaneously considers input and output data perturbations in all DMUs, namely, the change of the test DMU and the remaining DMUs. On the other hand, several authors investigated the sensitivity of DEA scores to the estimated efficiency frontier. For example, Simar and Wilson [7] [8] used a bootstrapping method to approximate the sampling distributions of DEA scores and to compute confidence intervals (CIs) for such scores. Barnum et al. [9] provided an alternative methodology based on Panel Data Analysis (PDA) for computing CIs of DEA scores; in sum, they complemented Simar and Wilson's bootstrapping by using panel data along with generalized least squares models to correct CIs for any violations of the standard statistical assumptions (i.e., DEA scores are independent and identically distributed, and normally distributed) such as the presence of contemporaneous correlation, serial correlation and heteroskedasticity. Note, however, that [7] and [8] do not take account of data variations in inputs and outputs. Note also that although [9] takes account of data variations in inputs and outputs by considering panel data and computing DEA scores separately for each cross section of the data, the reliability of the approach depends on the amount of data available for estimating the generalized least squares models.

In this paper, we follow the principles stated in Cook, Tone and Zhu [10] and believe that DEA performance measures are relative, not absolute, and frontiers-dependent. DEA scores undergo a change depending on the choice of inputs, outputs, DMUs and DEA models by which DMUs are evaluated. In this paper, we compute efficiency scores or equivalently solve the frontier problem using the non-oriented slacks-based super-efficiency model. Our approach deals with variations in both the estimated efficiency frontier and the input and output data directly by resampling from historical data over two different time frames (i.e., past-present and past-presentfuture); thus, the production possibility set for the entire DMUs differs with every sample ${ }^{1}$. In addition, our approach works for both small and large sets of data and does not make any parametric assumptions. Hence, our approach presents another alternative for computing confidence intervals of DEA scores.

This paper unfolds as follows. Section 2 presents a generic methodological framework to estimate the confidence intervals of DEA scores under a past-present time frame and extends it to the past-present-future time frame. Section 3 presents a healthcare application to illustrate the proposed resampling framework. Finally, section 5 concludes the paper.

\section{Proposed Methodology}

In this section, we propose a generic methodological framework to estimate the confidence intervals of DEA scores under a past-present time frame. This framework is generic in that its implementation requires a number of decisions to be made as will be discussed hereafter. Then, we extend the use of this framework to the pastpresent-future time frame.

\subsection{Past-Present Based Framework}

The first framework is designed for when past-present information on say $m$ inputs and $s$ outputs of a set of $n$ DMUs is available; that is, $\left(X^{t}, Y^{t}\right)=\left\{\left(x_{i, j}^{t}, y_{r, j}^{t}\right) ; i=1, \cdots, m, r=1, \cdots, s, j=1 \cdots, n,\right\}, t=1, \cdots, T$, where period $T$ denote the present and periods 1 though $T-1$ represent the past. The proposed framework could be summarized as follows:

\section{Initialization Step}

Choose an appropriate DEA model for computing the efficiency scores of DMUs;

Use the chosen DEA model to estimate the DEA scores of DMUs based on the present information; that is,

\footnotetext{
${ }^{1}$ Throughout this paper, we assume that the dataset is free from outliers and homogenous in the kind of DMUs (e.g., hospitals, banks or universities in the same category). For outlier detection, see Yang et al. [11] and references therein.
} 
$\left(X^{T}, Y^{T}\right)$. Let $\delta_{j}^{T} ; j=1, \cdots, n$ denote such scores-in the iterative step, we gauge the confidence interval of $\delta_{j}^{T} ; j=1, \cdots, n$ using replicas of historical data $\left(X^{t}, Y^{t}\right), t=1, \cdots, T$;

Choose an appropriate scheme, say $w$, to weigh the available information on the past and the present;

Choose a confidence level $1-\alpha$;

Choose the number of replicas or samples to draw from the past, say $B$, along with any properties they should satisfy before being considered appropriate to use for generating the sampling distributions of $\delta_{j}^{T} ; j=1, \cdots, n$ and computing their confidence intervals;

Set an indicator variable, say property_status, that reflects whether the $B$ replicas satisfy the required properties or not to false.

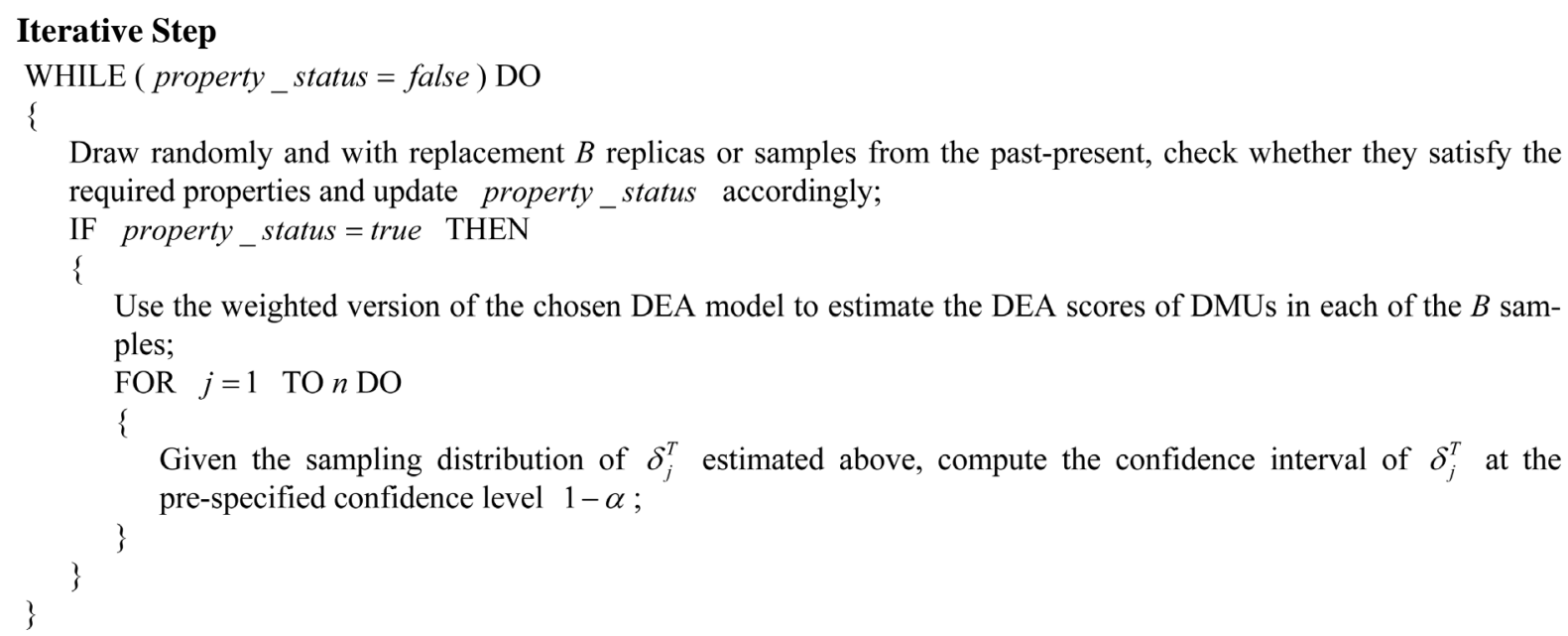

The generic nature of this framework requires a number of decisions to be made for its implementation for a particular application. Hereafter, we shall discuss how one might make such decisions.

\subsubsection{Choice of a DEA Model}

In principle one might choose from a relatively wide range of DEA models; however, given the nature of this exercise we recommend the use of the non-oriented super slacks-based measure model (Tone [12] and Ouenniche et al. [13]) under the relevant returns-to-scale (RTS) setup (e.g., constant, variable, increasing, decreasing) as suggested by the RTS analysis of the dataset one is dealing with. This model is an extension of the SBM (slacks-based measure) model of Tone [14]—see also [15]. Although one could use other models (e.g., radial or oriented), our recommendation is based on the following reasons. First, as a non-radial model, the SBM model is appropriate for taking account of input and output slacks which affect efficiency scores directly, whereas the radial models are mainly concerned with the proportional changes in inputs or outputs. Thus, SBM scores are more sensitive to data variations than the radial ones. Second, the non-oriented SBM model can deal with input-surpluses and output-shortfalls within the same scheme. Finally, as most DEA scores are bounded by unity ( $\leq 1$, or $\geq 1$ ), difficulties in comparing efficient DMUs maybe encountered; therefore, we recommend using the super-efficiency version of the non-oriented SBM as it removes such unity bounds.

\subsubsection{Choice of a Weighting Scheme for Past-Present Information}

Many different weighting schema could be used to weigh information on the past and the present; that is, $\left(x_{i, j}^{t}\right) ; i=1, \cdots, m, j=1, \cdots, n, t=1, \cdots, T$ and $\left(y_{r, j}^{t}\right) ; r=1, \cdots, s, j=1, \cdots, n, t=1, \cdots, T$. The choice of the weighting scheme should reflect the decision makers' perspective and knowledge of the application area on how the past should influence the present. In this paper, we set the weight $w_{t}$ of a period $t$ so that the weights are increasing in $\mathrm{t}$; in sum, we assume that more recent periods carry information that is more relevant to estimate efficiency scores in the present time. Thus, the following Lucas number series $\left(l_{1}, \cdots, l_{T}\right)$, a variant of Fibonacci series, is a candidate where $l_{t+2}=l_{t}+l_{t+1} ; t=1, \cdots, T-2, l_{1}=1, l_{2}=2$. Let $L$ denote the sum of the series: $L=\sum_{t=1}^{T} l_{t}$. We define weight $w_{t}$ as $l_{t} / L$ for $t=1, \cdots, T$. For example, when $T=5$, we have $w_{1}=0.0526$, 
$w_{2}=0.1053, w_{3}=0.1579, w_{4}=0.2631$, and $w_{5}=0.4211$. Thus, the influence of the past periods fades away gradually as we approach the present.

\subsubsection{Choice of the Replication Process and the Number of Replicas}

In this paper, we regard historical data $\left(X^{t}, Y^{t}\right)=\left\{\left(x_{i, j}^{t}, y_{r, j}^{t}\right) ; i=1, \cdots, m, r=1, \cdots, s, j=1, \cdots, n,\right\}, t=1, \cdots, T$ as discrete events with probability $w_{t}$ and cumulative probability $W_{t}=\sum_{k=1}^{t} w_{k} ; t=1, \cdots, T$. We propose a replication process based on bootstrapping. First proposed by Efron [16], nowadays bootstrapping refers to a collection of methods that randomly resample with replacement from the original sample. Thus, in bootstrapping, the population is to the sample what the sample is to the bootstrapped sample. Bootstrapping could be either parametric or non-parametric. Parametric bootstrapping is concerned with fitting a parametric model, which in our case would be a theoretical distribution, to the data and sampling from such fitted distribution. This is a viable approach for large datasets where the distribution of each input and each output could be reasonably approximated by a specific theoretical distribution. However, when no theoretical distribution could serve as a good approximation to the empirical one or when the dataset is small, non-parametric bootstrapping is the way to proceed. Non-parametric bootstrapping does not make any assumptions except that the sample distribution is a good approximation to the population distribution, or equivalently the sample is representative of the population. Consequently, datasets with different features require different resampling methods that take account of such features and thus generate representative replicas.

For a non-correlated and homoskedastic dataset, one could for example use smooth bootstrapping or Bayesian bootstrapping, where smooth bootstrapping generates replicas by adding small amounts of zero-centered random noise (usually normally distributed) to resampled observations, whereas Bayesian bootstrapping generates replicas by reweighting the initial data set according to a randomly generated weighting scheme. In this paper, we recommend the use of a variant of Bayesian bootstrapping whereby the weighting scheme consists of the Lucas number series-based weights $w_{t}$ presented above, because it is more appropriate when one is resampling over a past-present time frame and more recent information is considered more valuable. For a non-correlated and homoskedastic dataset, our Data Generation Process (DGP) may be summarized as follows. First, a random number $\rho$ is drawn from the uniform distribution over the interval [0,1], then whichever cross section data $\left(X^{t}, Y^{t}\right)$ so that $W_{t-1}<\rho \leq W_{t}$ is resampled, where $W_{0}=0$. This process is repeated as many times as necessary to produce the required number of valid replicas or samples.

On the other hand, for a correlated and/or heteroskedastic dataset, one could use one of the block bootstrapping methods, where replicas are generated by splitting the dataset into non-overlapping blocks (simple block bootstrap) or into overlapping blocks of the same or different lengths (moving block bootstrap), sampling such blocks with replacement and then aligning them in the order they were drawn. The main idea of all block bootstrap procedures consists of dividing the data into blocks of consecutive observations of length $\ell$, say $\left[\left(X^{t}, Y^{t}\right),\left(X^{t+1}, Y^{t+1}\right), \cdots,\left(X^{t+\ell-1}, Y^{t+\ell-1}\right)\right]$, and sampling the blocks randomly with replacement from all possible Blocks - for an overview of bootstrapping methods, the reader is referred to [17]. The block bootstrap procedure with blocks of non-random length can be summarized as follows:

Input: Block length $\ell \in \mathbb{N}$ so that $\ell \ll T$.

Step 1: Draw randomly and independently block labels, say $b_{1}, b_{2}, \cdots, b_{R+1}$, from the set of labels, say $L$, where $R=[T / \ell], L=\{1, \ell+1,2 \ell+1, \cdots,(R-1) \ell+1\}$ if non-overlapping blocks are considered, and $L=\{1,2, \cdots, T-\ell+1\}$ if overlapping blocks are considered.

Step 2: Lay the blocks $\left[\left(X^{b_{k}}, Y^{b_{k}}\right),\left(X^{b_{k}+1}, Y^{b_{k}+1}\right), \cdots,\left(X^{b_{k}+\ell-1}, Y^{b_{k}+\ell-1}\right)\right] ; k=1, \cdots, R+1$, end-to-end in the order sampled together and discard the last $\ell-T+R \ell$ observations to form a bootstrap series $\left(\hat{X}^{1}, \hat{Y}^{1}\right),\left(\hat{X}^{2}, \hat{Y}^{2}\right), \cdots,\left(\hat{X}^{T}, \hat{Y}^{T}\right)$.

Output: Bootstrap sample $\left(\hat{X}^{1}, \hat{Y}^{1}\right),\left(\hat{X}^{2}, \hat{Y}^{2}\right), \cdots,\left(\hat{X}^{T}, \hat{Y}^{T}\right)$.

As to the choice of the number of replicas $B$, there is no universal rule except that the larger the value of $B$ the more stable the results. However, one should take into consideration the computational requirements; therefore, in practice, one would keep increasing the value of $B$ until the simulation converges; that is, the results from a run do not change when adding more iterations. 


\subsubsection{Choice of the Properties the Replicas Should Satisfy}

As replicas are required to be representative of the dataset under consideration, one would have to perform a preliminary analysis of the data to find out about its features; namely, whether it is correlated or not and whether it is heteroskedastic or not using statistical tests such as the ones used in [9]. For a correlated and/or heteroskedastic dataset, the same relevant statistical tests would have to be used to find out whether the replicas are representative or not. When replicas are not representative, one would have to reject them and resample again. However, for a non-correlated and homoskedastic dataset, one could use hypothesis tests or confidence intervals based on Fisher's z transformation to compare correlation patterns in past and present data. For example, for the present time period data, one could compute the correlation coefficient between all pairs of inputs, outputs, and input-output over all DMUs. Then, compute their $\zeta \%$ confidence intervals; e.g., 95\%, using Fisher's z transformation [18]. If the corresponding correlation of a resampled data is out of range of this interval, we discard this resample data. Thus, inappropriate samples with unbalanced inputs and outputs relative to the inputs and outputs of the last period are excluded from resampling. The above noted $95 \%$ confidence interval is not compulsory. The narrower the interval, the closer the resample will be to the last period data.

\subsection{Past-Present-Future Time Based Framework}

In the previous subsection, we utilized historical data $\left(X^{t}, Y^{t}\right), t=1, \cdots, T$ to gauge the confidence interval of the last period's scores. In this section, we forecast the "future"; namely, $\left(X^{T+1}, Y^{T+1}\right)$ by using "past-present" data $\left(X^{t}, Y^{t}\right), t=1, \cdots, T$ and forecast the efficiency scores of the future DMUs along with their confidence intervals. In order to avoid repetition, hereafter we shall discuss how the past-present time based framework could be extended to the past-present-future context. First, we have to forecast the future; to be more specific, given the observed historical data $\left(x_{i, j}^{t}, y_{r, j}^{t}\right), t=1, \cdots, T$ for a certain input $i(i=1, \cdots, m)$ and output $r(r=1, \cdots, s)$ of a DMU $j(j=1, \cdots, n)$, we wish to forecast $\left(x_{i, j}^{T+1}, y_{r, j}^{T+1}\right)$. There are several forecasting engines available for this purpose. Once these forecasts are obtained, we then estimate the super-efficiency score of the "future" DMU $\left(X^{T+1}, Y^{T+1}\right)$ using the non-oriented super slacks-based measure model. Finally, given the past-presentfuture inter-temporal data set $\left(X^{t}, Y^{t}\right), t=1, \cdots, T+1$, we apply the resampling scheme proposed in the previous section and obtain confidence intervals.

\section{An Application in Healthcare}

In this study we utilize a dataset concerning nineteen Japanese municipal hospitals from 2007 to 2009 to illustrate how the proposed framework works. There are approximately 1000 municipal hospitals in Japan and there is large heterogeneity amongst them. We selected nineteen municipal hospitals with more than 400 beds. Therefore, this sample may represent larger acute-care hospitals with homogeneous functions. The data were collected from the Annual Databook of Local Public Enterprises published by the Ministry of Internal Affairs and Communications. For illustration purposes, we chose for this study two inputs; namely, Doctor ((I)Doc) and Nurse ((I)Nur), and two outputs; namely, Inpatient ((O)In) and Outpatient ((O)Out). Table 1 exhibits the data, while Table 2 shows the main statistics. The data are the yearly averages of the fiscal year data, as we have no daily or monthly data, and the Japanese government's fiscal year begins on April 1 and ends on March 31. As can be seen, the data on inputs and outputs fluctuate by year, which suggests the need for analysis of data variation.

We solved the non-oriented super slacks-based measure model year by year and obtained the super-efficiency scores in Table 3 along with their graphical representation in Figure 1. As can be seen, the scores fluctuate by year. Once again, this suggests the need for analysis of data variation. If we had daily data, this could be done. However, we only have fiscal-year data and hence we need to resample data in order to gauge the confidence interval of efficiency scores. Then, we merged the dataset of all years and evaluated the efficiency scores relative to $57(=19 \times 3)$ DMUs as exhibited in Table 4 and Figure 2. Comparing the averages of these three years, we found that the average 0.820 of year 2007 is better than $2008(0.763)$ and $2009(0.732)$. We also performed the non-parametric Wilcoxon rank-sum test and the results indicate that the null hypothesis; that is, 2007 and 2008 have the same distribution of efficiency scores, is rejected at the significance level 1\%; therefore, 2007 outperforms 2008. Similarly, 2007 outperforms 2009. However, we cannot see significant difference between 2008 and 2009. 
Table 1. The data.

\begin{tabular}{|c|c|c|c|c|c|c|c|c|c|c|c|c|}
\hline & 2007 & & & & 2008 & & & & 2009 & & & \\
\hline DMU & (I)Doc & (I)Nur & (O)In & (O)Out & (I)Doc & (I)Nur & (O)In & (O)Out & (I)Doc & (I)Nur & (O)In & (O)Out \\
\hline $\mathrm{H} 1$ & 108 & 433 & 606 & 1239 & 114 & 453 & 617 & 1244 & 116 & 545 & 603 & 1295 \\
\hline H2 & 125 & 448 & 642 & 1363 & 133 & 499 & 638 & 1310 & 136 & 482 & 618 & 1300 \\
\hline H3 & 118 & 567 & 585 & 1072 & 121 & 600 & 569 & 1051 & 125 & 616 & 561 & 1071 \\
\hline $\mathrm{H} 4$ & 138 & 541 & 699 & 1210 & 138 & 531 & 704 & 1194 & 140 & 554 & 679 & 1182 \\
\hline H5 & 138 & 613 & 653 & 1195 & 142 & 616 & 644 & 1147 & 137 & 633 & 622 & 1147 \\
\hline H6 & 99 & 569 & 716 & 1533 & 106 & 592 & 701 & 1478 & 109 & 613 & 651 & 1457 \\
\hline H7 & 94 & 498 & 540 & 1065 & 103 & 494 & 551 & 1067 & 101 & 491 & 540 & 1067 \\
\hline H8 & 106 & 461 & 496 & 1051 & 118 & 490 & 504 & 1033 & 133 & 479 & 505 & 1081 \\
\hline H9 & 109 & 450 & 483 & 851 & 119 & 483 & 487 & 877 & 121 & 501 & 486 & 904 \\
\hline H10 & 102 & 540 & 581 & 1268 & 106 & 558 & 565 & 1278 & 148 & 611 & 586 & 1321 \\
\hline H11 & 92 & 495 & 490 & 1217 & 101 & 497 & 501 & 1146 & 102 & 501 & 479 & 1113 \\
\hline H12 & 148 & 721 & 771 & 1637 & 147 & 710 & 723 & 1657 & 158 & 737 & 743 & 1714 \\
\hline H13 & 103 & 593 & 679 & 2011 & 106 & 673 & 642 & 1883 & 120 & 697 & 634 & 1872 \\
\hline H14 & 101 & 500 & 613 & 1868 & 110 & 519 & 617 & 1894 & 116 & 517 & 623 & 2009 \\
\hline H15 & 159 & 793 & 964 & 2224 & 160 & 801 & 906 & 2148 & 166 & 817 & 877 & 2155 \\
\hline H16 & 77 & 354 & 410 & 1047 & 68 & 359 & 391 & 916 & 81 & 378 & 406 & 897 \\
\hline H17 & 111 & 663 & 717 & 1674 & 112 & 645 & 702 & 1774 & 112 & 663 & 709 & 1733 \\
\hline H18 & 62 & 388 & 480 & 913 & 64 & 385 & 467 & 907 & 63 & 381 & 463 & 872 \\
\hline H19 & 98 & 323 & 508 & 1192 & 95 & 314 & 483 & 1018 & 95 & 320 & 490 & 1034 \\
\hline
\end{tabular}

Table 2. Main statistics.

\begin{tabular}{|c|c|c|c|c|c|c|c|c|c|c|c|c|}
\hline & 2007 & & & & 2008 & & & & 2009 & & & \\
\hline & (I)Doc & (I)Nur & (O)In & (O)Out & (I)Doc & (I)Nur & (O)In & (O)Out & (I)Doc & (I)Nur & (O)In & (O)Out \\
\hline Min & 62 & 323 & 410 & 851 & 64 & 314 & 391 & 877 & 63 & 320 & 406 & 872 \\
\hline Max & 159 & 793 & 964 & 2224 & 160 & 801 & 906 & 2148 & 166 & 817 & 877 & 2155 \\
\hline Avg & 110 & 524 & 612 & 1349 & 114 & 538 & 601 & 1317 & 120 & 555 & 593 & 1328 \\
\hline StdDev & 23.75 & 120.41 & 130.51 & 378.24 & 24.15 & 121.43 & 119.57 & 380.07 & 25.58 & 126.78 & 113.05 & 389.49 \\
\hline
\end{tabular}

\subsection{Illustration of the Past-Present Framework}

We applied the proposed procedure to the historical data of nineteen hospitals for the two years 2008-2009 in Table 1. We excluded the year 2007 data, because they belong to a different population than 2009 as explained in Preliminary results (Panel). Note that historical data may suffer from accidental or exceptional events, for example, oil shock, earthquake, financial crisis, environmental system change and so forth. We must exclude these from the data. If some data are under age depreciation, we must adjust them properly. In this study, we use Lucas weights for past and present data. However, we can use other weighting schema (e.g., exponential) as well.

Table 5 shows the correlation matrix of the observed 2009 year data in Table 1 and Fisher 95\% confidence intervals are exhibited in Table 6. For example, the correlation coefficient between Doc and Outpatient is 
Table 3. Super-SBM scores by cross section (year).

\begin{tabular}{llll}
\hline & 2007 & 2008 & 2009 \\
\hline H1 & 0.883 & 0.905 & 0.754 \\
H2 & 0.875 & 0.801 & 0.779 \\
H3 & 0.623 & 0.615 & 0.592 \\
H4 & 0.700 & 0.765 & 0.680 \\
H5 & 0.619 & 0.620 & 0.604 \\
H6 & 1.004 & 0.942 & 0.848 \\
H7 & 0.719 & 0.732 & 0.725 \\
H8 & 0.676 & 0.651 & 0.631 \\
H9 & 0.588 & 0.583 & 0.568 \\
H10 & 0.758 & 0.764 & 0.631 \\
H11 & 0.757 & 0.740 & 0.698 \\
H12 & 0.711 & 0.741 & 0.714 \\
H13 & 1.034 & 1.025 & 0.831 \\
H14 & 1.039 & 1.107 & 1.145 \\
H15 & 0.858 & 0.857 & 0.811 \\
H16 & 0.831 & 0.847 & 0.742 \\
H17 & 0.847 & 0.948 & 0.937 \\
H18 & 1.034 & 1.050 & 1.074 \\
H19 & 1.071 & 0.072 & 0.782 \\
\hline
\end{tabular}

Table 4. Super-SBM scores for panel data (all years).

\begin{tabular}{|c|c|c|c|}
\hline & 2007 & 2008 & 2009 \\
\hline H1 & 0.883 & 0.833 & 0.727 \\
\hline H2 & 0.875 & 0.750 & 0.745 \\
\hline H3 & 0.623 & 0.584 & 0.571 \\
\hline $\mathrm{H} 4$ & 0.700 & 0.712 & 0.654 \\
\hline H5 & 0.619 & 0.590 & 0.584 \\
\hline H6 & 1.004 & 0.860 & 0.783 \\
\hline H7 & 0.719 & 0.696 & 0.699 \\
\hline H8 & 0.676 & 0.620 & 0.613 \\
\hline H9 & 0.588 & 0.556 & 0.551 \\
\hline H10 & 0.758 & 0.726 & 0.610 \\
\hline H11 & 0.757 & 0.703 & 0.672 \\
\hline H12 & 0.711 & 0.704 & 0.688 \\
\hline H13 & 1.034 & 0.871 & 0.794 \\
\hline H14 & 1.024 & 0.950 & 1.020 \\
\hline H15 & 0.858 & 0.812 & 0.779 \\
\hline H16 & 0.831 & 0.798 & 0.715 \\
\hline H17 & 0.847 & 0.872 & 0.855 \\
\hline H18 & 1.028 & 0.929 & 0.922 \\
\hline H19 & 1.042 & 0.920 & 0.924 \\
\hline Avg. & 0.820 & 0.763 & 0.732 \\
\hline
\end{tabular}


Table 5. Correlation matrix.

\begin{tabular}{ccccc}
\hline & Doc & Nurse & Inpatient & Outpatient \\
\hline Doc & 1 & 0.7453 & 0.7372 & 0.5178 \\
Nurse & 0.7453 & 1 & 0.8610 & 0.7387 \\
Inpatient & 0.7372 & 0.8610 & 1 & 0.8264 \\
Outpatient & 0.5178 & 0.7387 & 0.8264 & 1 \\
\hline
\end{tabular}

Table 6. Fisher 95\% confidence lower/upper bounds for correlation matrix.

\begin{tabular}{|c|c|c|c|c|c|}
\hline & & \multicolumn{4}{|c|}{ Lower bounds } \\
\hline & & Doc & Nurse & Inpatient & Outpatient \\
\hline & Doc & & 0.4400 & 0.4255 & 0.0832 \\
\hline Upper & Nurse & 0.8961 & & 0.6681 & 0.4281 \\
\hline \multirow[t]{2}{*}{ bounds } & Inpatient & 0.8926 & 0.9455 & & 0.5959 \\
\hline & Outpatient & 0.7869 & 0.8932 & 0.9311 & \\
\hline
\end{tabular}

- $2007 \quad 2008-2009$

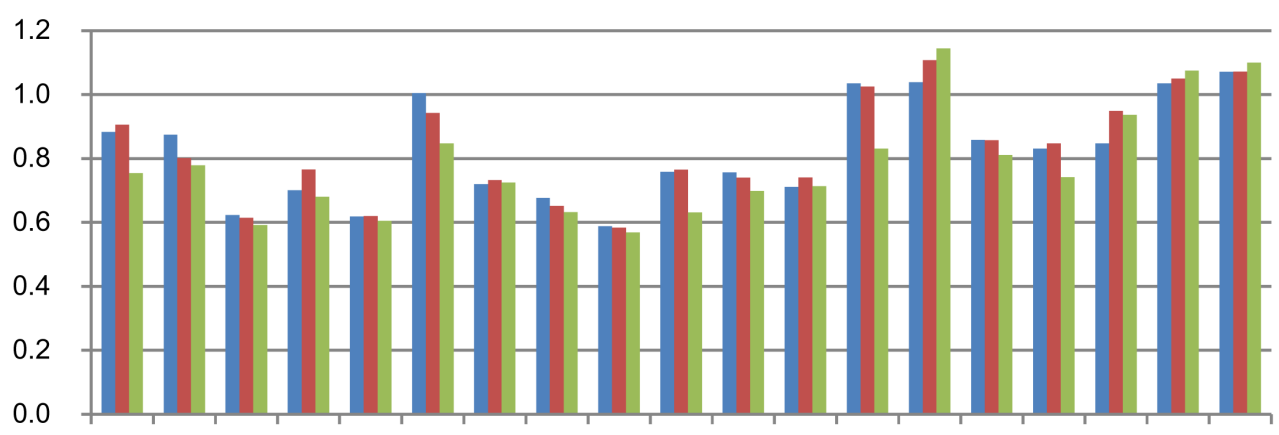

$\begin{array}{lllllllllllllllllllllll} & \mathrm{H} 1 & \mathrm{H} 2 & \mathrm{H} 3 & \mathrm{H} 4 & \mathrm{H} 5 & \mathrm{H} 6 & \mathrm{H} 7 & \mathrm{H} 8 & \mathrm{H} 9 & \mathrm{H} 10 & \mathrm{H} 11 & \mathrm{H} 12 & \mathrm{H} 13 & \mathrm{H} 14 & \mathrm{H} 15 & \mathrm{H} 16 & \mathrm{H} 17 & \mathrm{H} 18 & \mathrm{H} 19\end{array}$

Figure 1. Super-SBM scores by cross section (year).

ロ $2007 \quad 2008 \quad 2009$

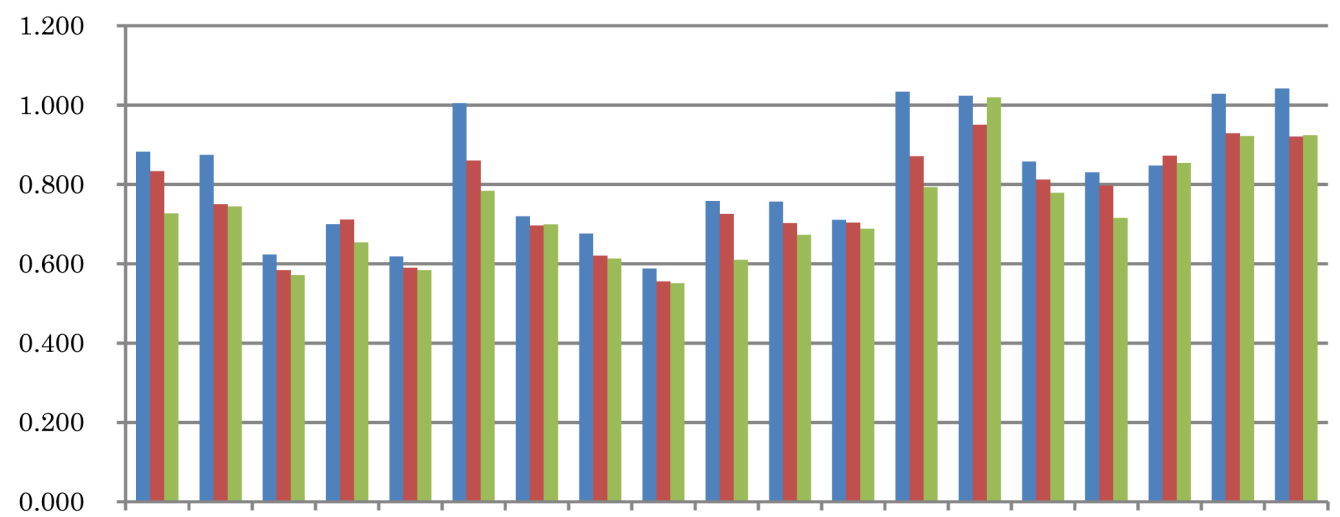

H1 H2 H3 $\quad$ H4 $\quad$ H5 $\quad$ H6 $\quad$ H7 $\quad$ H8 $\quad$ H9 H10 H11 H12 H13 H14 H15 H16 H17 H18 H19

Figure 2. Super-SBM Scores for panel data (all years). 
0.5178 and its 95\% lower/upper bounds are respectively 0.0832 and 0.7869 . In addition, we report Fisher 20\% confidence lower/upper bounds in Table 7 . The intervals are considerably narrowed down compared with Fisher $95 \%$ case.

Table 8 exhibits results obtained by 500 replicas where the column DEA is the last period's (2009) efficiency score and Average indicates the average score over 500 replicas. The column Rank is the ranking of average scores. We applied Fisher 95\% threshold and found no out-of-range samples. Figure 3 shows the 95\% confidence intervals for the last period's (2009) DEA scores along with Average scores. The average of the 95\% confidence interval for all hospitals is 0.10 .

In the Fisher 95\% (ל95) case, we found no discarded samples, whereas in the Fisher 20\% (ל20) case, 1945 samples were discarded before getting 500 replicas. Table 9 shows the comparisons of scores calculated by both thresholds, where we cannot see significant differences.

Note that one resample produces one efficiency score for each DMU. We compared 500 and 5000 replicas and obtained the $95 \%$ confidence interval as exhibited in Table 10. As can be seen, the difference is negligibly small. 500 replicas may be acceptable in this case. However, the number of replicas depends on the numbers of

Table 7. Fisher $20 \%$ confidence lower/upper bounds for correlation matrix.

\begin{tabular}{cccccc}
\hline & & \multicolumn{3}{c}{ Lower bounds } \\
\cline { 3 - 6 } & & Doc & Nurse & Inpatient & Outpatient \\
\hline \multirow{2}{*}{ Upper } & Doc & - & 0.71578 & 0.70695 & 0.46998 \\
bounds & Nurse & 0.77214 & - & 0.8437 & 0.70854 \\
& Inpatient & 0.76482 & 0.87652 & - & 0.80525 \\
& Outpatient & 0.56266 & 0.76614 & 0.84547 & - \\
\hline
\end{tabular}

Table 8. DEA score and confidence interval with 500 replicas.

\begin{tabular}{|c|c|c|c|c|c|}
\hline & $97.50 \%$ & DEA (2009) & Average & $2.50 \%$ & Rank (Avg) \\
\hline $\mathrm{H} 1$ & 0.9228 & 0.754 & 0.8047 & 0.724 & 8 \\
\hline $\mathrm{H} 2$ & 0.8279 & 0.7787 & 0.7865 & 0.7415 & 9 \\
\hline H3 & 0.6285 & 0.5918 & 0.5999 & 0.573 & 18 \\
\hline $\mathrm{H} 4$ & 0.7574 & 0.6802 & 0.709 & 0.6694 & 14 \\
\hline H5 & 0.6375 & 0.6042 & 0.6088 & 0.5792 & 17 \\
\hline H6 & 0.9384 & 0.8475 & 0.8758 & 0.8159 & 6 \\
\hline H7 & 0.762 & 0.725 & 0.7284 & 0.6998 & 11 \\
\hline H8 & 0.6902 & 0.6311 & 0.6365 & 0.6002 & 16 \\
\hline H9 & 0.603 & 0.5681 & 0.5732 & 0.5452 & 19 \\
\hline H10 & 0.7963 & 0.6308 & 0.6818 & 0.6032 & 15 \\
\hline H11 & 0.7433 & 0.6985 & 0.7116 & 0.6808 & 13 \\
\hline H12 & 0.7684 & 0.714 & 0.7237 & 0.6849 & 12 \\
\hline H13 & 1.0465 & 0.831 & 0.8978 & 0.8081 & 5 \\
\hline H14 & 1.1564 & 1.1448 & 1.1329 & 1.1037 & 1 \\
\hline H15 & 0.8692 & 0.8107 & 0.8277 & 0.7886 & 7 \\
\hline H16 & 0.8792 & 0.7418 & 0.7782 & 0.714 & 10 \\
\hline H17 & 1.0142 & 0.9368 & 0.9542 & 0.9076 & 4 \\
\hline H18 & 1.0837 & 1.0745 & 1.0708 & 1.0497 & 3 \\
\hline H19 & 1.1194 & 1.0996 & 1.0897 & 1.0618 & 2 \\
\hline
\end{tabular}


Table 9. Comparisons of Fisher's 20\% (ל20) and 95\% (५95) thresholds.

\begin{tabular}{ccccccccccc}
\hline & & & & & & & & $\zeta 20-\zeta 95$ & $\zeta 20-\zeta 95$ \\
\hline 520 & $97.50 \%$ & DEA & $2.50 \%$ & $\zeta 95$ & $97.50 \%$ & DEA & $2.50 \%$ & $97.50 \%$ & $2.50 \%$ \\
\hline H1 & 0.9061 & 0.754 & 0.724 & H1 & 0.9228 & 0.754 & 0.724 & -0.017 & 0.000 \\
H2 & 0.8247 & 0.7787 & 0.7419 & H2 & 0.8279 & 0.7787 & 0.7415 & -0.003 & 0.000 \\
H3 & 0.6279 & 0.5918 & 0.5757 & H3 & 0.6285 & 0.5918 & 0.573 & -0.001 & 0.003 \\
H4 & 0.7476 & 0.6802 & 0.6684 & H4 & 0.7574 & 0.6802 & 0.6694 & -0.010 & -0.001 \\
H5 & 0.6375 & 0.6042 & 0.5832 & H5 & 0.6375 & 0.6042 & 0.5792 & 0.000 & 0.004 \\
H6 & 0.9382 & 0.8475 & 0.8168 & H6 & 0.9384 & 0.8475 & 0.8159 & 0.000 & 0.001 \\
H7 & 0.7611 & 0.725 & 0.6989 & H7 & 0.762 & 0.725 & 0.6998 & -0.001 & -0.001 \\
H8 & 0.6905 & 0.6311 & 0.6011 & H8 & 0.6902 & 0.6311 & 0.6002 & 0.000 & 0.001 \\
H9 & 0.6023 & 0.5681 & 0.5467 & H9 & 0.603 & 0.5681 & 0.5452 & -0.001 & 0.001 \\
H10 & 0.7903 & 0.6308 & 0.6044 & H10 & 0.7963 & 0.6308 & 0.6032 & -0.006 & 0.001 \\
H11 & 0.7469 & 0.6985 & 0.6808 & H11 & 0.7433 & 0.6985 & 0.6808 & 0.004 & 0.000 \\
H12 & 0.767 & 0.714 & 0.6828 & H12 & 0.7684 & 0.714 & 0.6849 & -0.001 & -0.002 \\
H13 & 1.0445 & 0.831 & 0.8081 & H13 & 1.0465 & 0.831 & 0.8081 & -0.002 & 0.000 \\
H14 & 1.1568 & 1.1448 & 1.1041 & H14 & 1.1564 & 1.1448 & 1.1037 & 0.000 & 0.000 \\
H15 & 0.867 & 0.8107 & 0.7886 & H15 & 0.8692 & 0.8107 & 0.7886 & -0.002 & 0.000 \\
H16 & 0.8747 & 0.7418 & 0.7222 & H16 & 0.8792 & 0.7418 & 0.714 & -0.004 & 0.008 \\
H17 & 1.0121 & 0.9368 & 0.9058 & H17 & 1.0142 & 0.9368 & 0.9076 & -0.002 & -0.002 \\
H18 & 1.0837 & 1.0745 & 1.0491 & H18 & 1.0837 & 1.0745 & 1.0497 & 0.000 & -0.001 \\
H19 & 1.1195 & 1.0996 & 1.063 & H19 & 1.1194 & 1.0996 & 1.0618 & 0.000 & 0.001 \\
\hline
\end{tabular}

Table 10. Comparisons of 5000 and 500 replicas (Fisher 95\%).

\begin{tabular}{|c|c|c|c|c|c|c|c|c|c|c|}
\hline \multicolumn{4}{|c|}{500 Replica } & \multicolumn{4}{|c|}{5000 Replica } & & \multicolumn{2}{|c|}{ Difference } \\
\hline 500 & $97.50 \%$ & DEA & $2.50 \%$ & 5000 & $97.50 \%$ & DEA & $2.50 \%$ & & $97.50 \%$ & $2.50 \%$ \\
\hline $\mathrm{H} 1$ & 0.9228 & 0.754 & 0.724 & $\mathrm{H} 1$ & 0.9184 & 0.754 & 0.7227 & & 0.0044 & 0.0013 \\
\hline $\mathrm{H} 2$ & 0.8279 & 0.7787 & 0.7415 & $\mathrm{H} 2$ & 0.8266 & 0.7787 & 0.7412 & & 0.0013 & 0.0003 \\
\hline H3 & 0.6285 & 0.5918 & 0.573 & H3 & 0.6291 & 0.5918 & 0.5719 & & -0.0006 & 0.0011 \\
\hline $\mathrm{H} 4$ & 0.7574 & 0.6802 & 0.6694 & $\mathrm{H} 4$ & 0.7581 & 0.6802 & 0.6679 & & -0.0007 & 0.0015 \\
\hline H5 & 0.6375 & 0.6042 & 0.5792 & H5 & 0.6379 & 0.6042 & 0.5801 & & -0.0004 & -0.0009 \\
\hline H6 & 0.9384 & 0.8475 & 0.8159 & H6 & 0.9423 & 0.8475 & 0.8164 & & -0.0039 & -0.0005 \\
\hline H7 & 0.762 & 0.725 & 0.6998 & H7 & 0.7615 & 0.725 & 0.6985 & & 0.0005 & 0.0013 \\
\hline H8 & 0.6902 & 0.6311 & 0.6002 & H8 & 0.6907 & 0.6311 & 0.5998 & & -0.0005 & 0.0004 \\
\hline H9 & 0.603 & 0.5681 & 0.5452 & H9 & 0.603 & 0.5681 & 0.5456 & & 0 & -0.0004 \\
\hline $\mathrm{H} 10$ & 0.7963 & 0.6308 & 0.6032 & H10 & 0.7942 & 0.6308 & 0.6055 & & 0.0021 & -0.0023 \\
\hline H11 & 0.7433 & 0.6985 & 0.6808 & H11 & 0.7447 & 0.6985 & 0.6808 & & -0.0014 & 0 \\
\hline H12 & 0.7684 & 0.714 & 0.6849 & $\mathrm{H} 12$ & 0.7684 & 0.714 & 0.6828 & & 0 & 0.0021 \\
\hline H13 & 1.0465 & 0.831 & 0.8081 & H13 & 1.046 & 0.831 & 0.8081 & & 0.0005 & 0 \\
\hline H14 & 1.1564 & 1.1448 & 1.1037 & H14 & 1.1565 & 1.1448 & 1.1026 & & $-1 \mathrm{E}-04$ & 0.0011 \\
\hline H15 & 0.8692 & 0.8107 & 0.7886 & H15 & 0.8726 & 0.8107 & 0.7886 & & -0.0034 & 0 \\
\hline H16 & 0.8792 & 0.7418 & 0.714 & H16 & 0.8785 & 0.7418 & 0.7198 & & 0.0007 & -0.0058 \\
\hline H17 & 1.0142 & 0.9368 & 0.9076 & H17 & 1.0141 & 0.9368 & 0.9051 & & $1 E-04$ & 0.0025 \\
\hline H18 & 1.0837 & 1.0745 & 1.0497 & H18 & 1.0837 & 1.0745 & 1.0459 & & 0 & 0.0038 \\
\hline \multirow[t]{3}{*}{ H19 } & 1.1194 & 1.0996 & 1.0618 & H19 & 1.1193 & 1.0996 & 1.0618 & & $1 \mathrm{E}-04$ & 0 \\
\hline & & & & & & & & Max & 0.0044 & 0.0038 \\
\hline & & & & & & & & Min & -0.0039 & -0.0058 \\
\hline
\end{tabular}




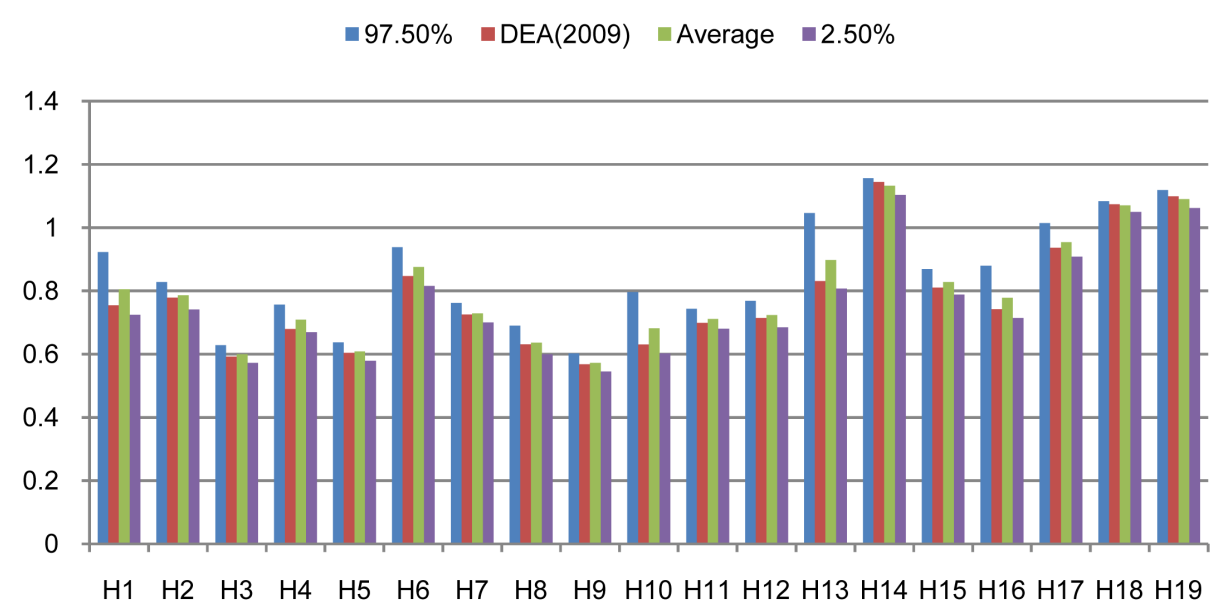

Figure 3. 95\% confidence interval.

inputs, outputs and DMUs. Hence, we need to check the variations of scores by increasing the number of replicas.

As to the comparisons of individual hospitals, looking at Hospitals 1 and 2 in Table 8 and Figure 3, we are puzzled which hospital exhibits better performance. Actually, the 2009 score and the Average score are reversed $(\mathrm{H} 1-2009=0.754, \mathrm{H} 1$-Average $=0.8047, \mathrm{H} 2-2009=0.7789, \mathrm{H} 2$-Average $=0.7865)$ and confidence intervals are overlapped. We applied the Wilcoxon rank-sum test and found that Hospital 1 outperforms Hospital 2 at the significant level 1\%. In this way, we can compare individual hospitals in efficiency measurements.

Finally, we would like to draw the reader's attention to the fact that, in some applications, one might set weights to inputs and outputs. Actually, if costs for inputs and incomes from outputs are available, we can evaluate the comparative cost performance of DMUs. In the absence of such information, instead, we can set weights to inputs and outputs. For example, the weights to Doc and Nurse are assumed to be 5 to 1 (on average), and those of Outpatient to Inpatient are 1 to 10 (on average). We can solve this problem via the Weighted-SBM model, which will enhance the reliability and applicability of our approach.

\subsection{Illustration of the Past-Present-Future Framework}

Hereafter, we shall present numerical results for the past-resent-future framework. In this case we regard 20072008 as the past-present and 2009 as the future. In our application, we used three simple prediction models to forecast the future; namely, a linear trend analysis model, a weighted average model with Lucas weights, and a hybrid model that consists of averaging their predictions.

Table 11 reports the forecasts for 2009 obtained by the linear trend analysis model. Table 12 shows the forecast DEA score and confidence interval along with the actual super-SBM score for 2009. Figure 4 exhibits 97.5\% percent, $2.5 \%$ percent, forecast score and actual score. It is observed that, of the nineteen hospitals, the actual 2009 scores of sixteen are included in the 95\% confidence interval. The average of Forecast-Actual over the nineteen hospitals was 0.063 (6.3\%).

Table 13 reports 2009 forecasts by the weighted average model with Lucas weights and Table 14 shows the actuals and the forecasts of 2009 scores along with confidence intervals. In this case, only four hospitals are included in the 95\% confidence interval. The average of Forecast-Actual over the nineteen hospitals was 0.056 (5.6\%). Although we did not report the results by the Average of Trend and Lucas case, the results are similar to the Lucas case. We compare the number of fails for the three forecast models that actual score is out of $97.5 \%$ and $2.5 \%$ interval. We have results as exhibited in Table 15. "Trend" gives the best performance among the three in this example.

\section{Conclusion}

DEA, originated by Charnes and Cooper (Charnes et al. [1]), is a non-parametric mathematical programming methodology that deals directly with input/output data. Using the data, DEA can evaluate the relative efficiency 
Table 11. 2009 forecasts: linear trend model.

\begin{tabular}{|c|c|c|c|c|}
\hline DMU & (I)Doc & (I)Nurse & (O)Inpatient & (O)Outpatient \\
\hline $\mathrm{H} 1$ & 120 & 473 & 628 & 1249 \\
\hline $\mathrm{H} 2$ & 141 & 550 & 634 & 1257 \\
\hline H3 & 124 & 633 & 553 & 1030 \\
\hline $\mathrm{H} 4$ & 138 & 521 & 709 & 1178 \\
\hline H5 & 146 & 619 & 635 & 1099 \\
\hline H6 & 113 & 615 & 686 & 1423 \\
\hline $\mathrm{H} 7$ & 112 & 490 & 562 & 1069 \\
\hline H8 & 130 & 519 & 512 & 1015 \\
\hline H9 & 129 & 516 & 491 & 903 \\
\hline $\mathrm{H} 10$ & 110 & 576 & 549 & 1288 \\
\hline H11 & 110 & 499 & 512 & 1075 \\
\hline H12 & 146 & 699 & 675 & 1677 \\
\hline H13 & 109 & 753 & 605 & 1755 \\
\hline H14 & 119 & 538 & 621 & 1920 \\
\hline H15 & 161 & 809 & 848 & 2072 \\
\hline H16 & 59 & 364 & 372 & 785 \\
\hline H17 & 113 & 627 & 687 & 1874 \\
\hline H18 & 66 & 382 & 454 & 901 \\
\hline H19 & 92 & 305 & 458 & 844 \\
\hline
\end{tabular}

Table 12. Forecast DEA score, actual (2009) score and confidence interval: forecast by linear trend model.

\begin{tabular}{|c|c|c|c|c|}
\hline DMU & $97.50 \%$ & Forecast (2009) & Actual (2009) & $2.50 \%$ \\
\hline H1 & 1.0237 & 0.9338 & 0.754 & 0.8245 \\
\hline $\mathrm{H} 2$ & 1.0027 & 0.787 & 0.7787 & 0.722 \\
\hline H3 & 0.6649 & 0.6148 & 0.5918 & 0.5641 \\
\hline $\mathrm{H} 4$ & 0.8816 & 0.8581 & 0.6802 & 0.7319 \\
\hline H5 & 0.6814 & 0.6421 & 0.6042 & 0.5771 \\
\hline H6 & 1.0213 & 0.8768 & 0.8475 & 0.8062 \\
\hline $\mathrm{H} 7$ & 0.8292 & 0.7586 & 0.725 & 0.6945 \\
\hline H8 & 0.7641 & 0.6725 & 0.6311 & 0.6066 \\
\hline H9 & 0.6983 & 0.6213 & 0.5681 & 0.539 \\
\hline $\mathrm{H} 10$ & 0.8422 & 0.7781 & 0.6308 & 0.7111 \\
\hline H11 & 0.8425 & 0.7206 & 0.6985 & 0.6679 \\
\hline H12 & 0.8136 & 0.7716 & 0.714 & 0.7068 \\
\hline H13 & 1.0814 & 1 & 0.831 & 0.8276 \\
\hline H14 & 1.1575 & 1.0909 & 1.1448 & 1.0281 \\
\hline H15 & 0.9467 & 0.8541 & 0.8107 & 0.7902 \\
\hline H16 & 1.0376 & 0.9444 & 0.7418 & 0.7258 \\
\hline H17 & 1.0387 & 1.0348 & 0.9368 & 0.8982 \\
\hline H18 & 1.0899 & 1.0537 & 1.0745 & 0.9692 \\
\hline H19 & 1.1354 & 1.0594 & 1.0996 & 1.0113 \\
\hline
\end{tabular}


Table 13. 2009 forecasts: Lucas weighted average model.

\begin{tabular}{|c|c|c|c|c|}
\hline DMU & (I)Doc & (I)Nurse & (O)Inpatient & (O)Outpatient \\
\hline $\mathrm{H} 1$ & 112 & 446 & 613 & 1242 \\
\hline $\mathrm{H} 2$ & 130 & 482 & 639 & 1328 \\
\hline H3 & 120 & 589 & 574 & 1058 \\
\hline $\mathrm{H} 4$ & 138 & 534 & 702 & 1199 \\
\hline H5 & 141 & 615 & 647 & 1163 \\
\hline H6 & 104 & 584 & 706 & 1496 \\
\hline $\mathrm{H} 7$ & 100 & 495 & 547 & 1066 \\
\hline H8 & 114 & 480 & 501 & 1039 \\
\hline H9 & 116 & 472 & 486 & 868 \\
\hline H10 & 105 & 552 & 570 & 1275 \\
\hline H11 & 98 & 496 & 497 & 1170 \\
\hline H12 & 147 & 714 & 739 & 1650 \\
\hline H13 & 105 & 646 & 654 & 1926 \\
\hline H14 & 107 & 513 & 616 & 1885 \\
\hline H15 & 160 & 798 & 925 & 2173 \\
\hline H16 & 71 & 357 & 397 & 960 \\
\hline H17 & 112 & 651 & 707 & 1741 \\
\hline H18 & 63 & 386 & 471 & 909 \\
\hline H19 & 96 & 317 & 491 & 1076 \\
\hline
\end{tabular}

Table 14. DEA score and confidence interval forecasts: Lucas weighted average model.

\begin{tabular}{lcccc}
\hline & $97.50 \%$ & Forecast (2009) & Actual (2009) & $2.50 \%$ \\
\hline H1 & 1.0001 & 0.8974 & 0.754 & 0.8469 \\
H2 & 0.9329 & 0.8527 & 0.7787 & 0.797 \\
H3 & 0.6448 & 0.6218 & 0.5918 & 0.5987 \\
H4 & 0.7855 & 0.7618 & 0.6802 & 0.7303 \\
H5 & 0.6584 & 0.64 & 0.6042 & 0.62 \\
H6 & 1.0101 & 0.9604 & 0.8475 & 0.7006 \\
H7 & 0.7347 & 0.6596 \\
H8 & 0.7813 & 0.6867 & 0.725 & 0.5894 \\
H9 & 0.7201 & 0.6177 & 0.6311 & 0.7441 \\
H10 & 0.7829 & 0.5681 & 0.7171 \\
H11 & 0.6578 & 0.6308 & 0.712 \\
H12 & 0.8109 & 0.7573 & 0.6985 & 1 \\
H13 & 0.8101 & 0.7336 & 0.714 & 1.0409 \\
H14 & 0.7623 & 1.0286 & 0.831 & 0.8263 \\
H15 & 1.059 & 1.0868 & 1.1448 & 0.7869 \\
H16 & 1.1306 & 0.8665 & 0.8107 & 0.8984 \\
H17 & 0.912 & 0.8488 & 0.7418 & 1.0115 \\
H18 & 0.9296 & 0.9427 & 0.9368 & 1.0745 \\
\hline
\end{tabular}


Table 15. Number of fails.

\begin{tabular}{cccc}
\hline & Trend & Lucas & Average of Trend and Lucas \\
\hline No. of fails & 3 & 15 & 15 \\
\hline
\end{tabular}

$\square 97.50 \% \quad \square \mathrm{DEA}($ Forecast $) \quad$ DEA(Actual) $\quad 2.50 \%$

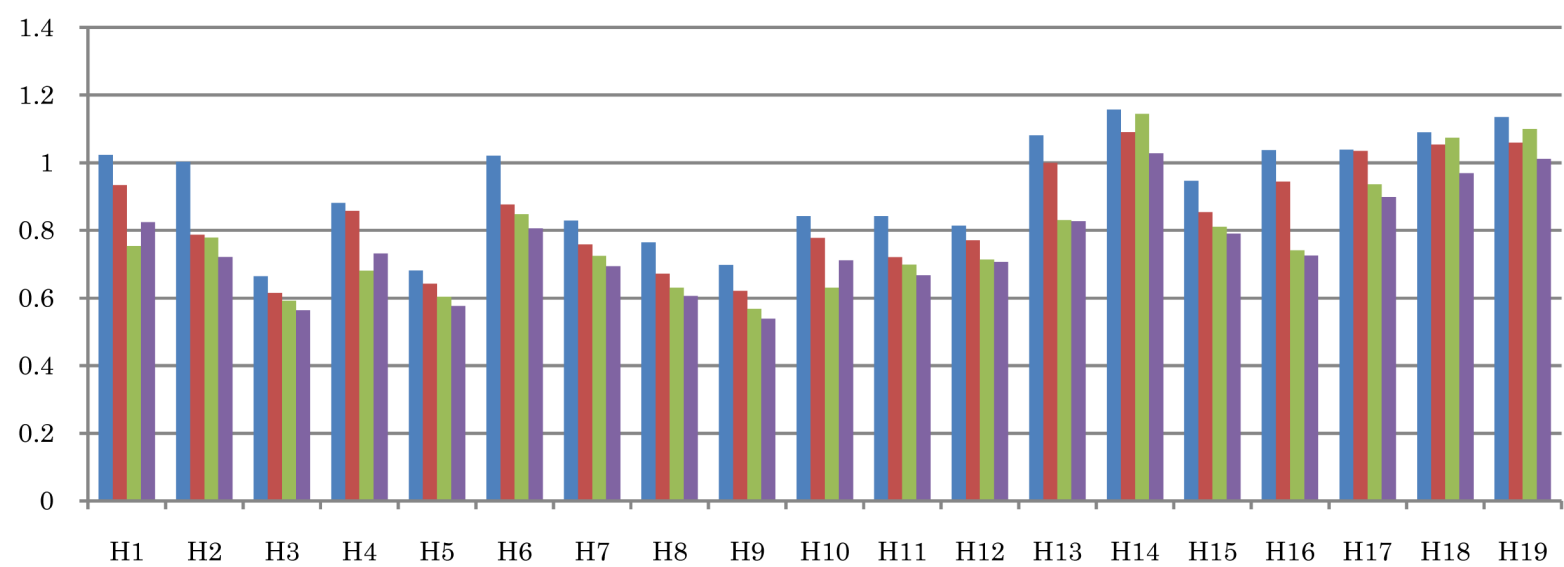

Figure 4. Confidence interval, forecast score and actual 2009 score: forecast by linear trend model.

of DMUs and propose a plan to improve the inputs/outputs of inefficient DMUs. This function is difficult to achieve with similar models in statistics, e.g., stochastic frontier analysis. DEA scores are not absolute but relative. They depend on the choice of inputs, outputs and DMUs as well as on the choice of model for assessing DMUs. DEA scores are subject to change and thus data variations in DEA should be taken into account. This subject should be discussed from the perspective of the itemized input/output variations. From this point of view, we have proposed two models. The first model utilizes historical data for the data generation process, and hence this model resamples data from a discrete distribution. It is expected that, if the historical data are volatile widely, confidence intervals will prove to be very wide, even when the Lucas weights are decreasing depending on the past-present periods. In such cases, application of the moving-average method is recommended. Rolling simulations will be useful for deciding on the choice of the length of the historical span. However, too many past year data are not recommended, because environments, such as healthcare service systems, are changing rapidly. The second model aims to forecast the future efficiency and its confidence interval. For forecasting, we used three models; namely, the linear trend model, the weighted average, and their average. On this subject, Xu and Ouenniche [19] [20] will be useful for the selection of forecasting models, and Chang et al. [21] will provide useful information on the estimation of the pessimistic and optimistic probabilities of the forecast of future input/output values.

\section{References}

[1] Charnes, A., Cooper, W.W. and Rhodes, E. (1978) Measuring the Efficiency of Decision Making Units. European Journal of Operational Research, 2, 429-444. http://dx.doi.org/10.1016/0377-2217(78)90138-8

[2] Banker, R. (1993) Maximum Likelihood, Consistency and Data Envelopment Analysis: A Statistical Foundation. Management Science, 39, 1265-1273. http://dx.doi.org/10.1287/mnsc.39.10.1265

[3] Banker, R. and Natarajan, R. (2004) Chapter 11. Statistical Test Based on DEA Efficiency Scores. In: Cooper, Seiford and Zhu, Eds., Handbook on Data Envelopment Analysis, Springer, 299-321. http://dx.doi.org/10.1007/1-4020-7798-x_11

[4] Charnes, A and Neralić, L. (1990) Sensitivity Analysis of the Additive Model in Data Envelopment Analysis. European Journal of Operational Research, 48, 332-341. http://dx.doi.org/10.1016/0377-2217(90)90416-9

[5] Neralić, L. (1998) Sensitivity Analysis in Models of Data Envelopment Analysis. Mathematical Communications, 3, 41-59. 
[6] Zhu, J. (2001) Super-Efficiency and DEA Sensitivity Analysis. European Journal of Operational Research, 129, 443-455. http://dx.doi.org/10.1016/S0377-2217(99)00433-6

[7] Simar, L. and Wilson, P. (1998) Sensitivity of Efficiency Scores: How to Bootstrap in Nonparametric Frontier Models. Management Science, 44, 49-61. http://dx.doi.org/10.1287/mnsc.44.1.49

[8] Simar, L. and Wilson, P.W. (2000) A General Methodology for Bootstrapping in Non-Parametric Frontier Models. Journal of Applied Statistics, 27, 779-802. http://dx.doi.org/10.1080/02664760050081951

[9] Barnum, D.T., Gleason, J.M., Karlaftis, M.G., Schumock, G.T., Shields, K.L., Tandon, S. and Walton, S.M. (2011) Estimating DEA Confidence Intervals with Statistical Panel Data Analysis. Journal of Applied Statistics, 39, 815-828. http://dx.doi.org/10.1080/02664763.2011.620948

[10] Cook, W.D., Tone, K. and Zhu, J. (2014) Data Envelopment Analysis: Prior to Choosing a Model. Omega, 44, 1-4. http://dx.doi.org/10.1016/j.omega.2013.09.004

[11] Yang, M., Wan, G. and Zheng, E. (2014) A Predictive DEA Model for Outlier Detection. Journal of Management Analytics, 1, 20-41. http://dx.doi.org/10.1080/23270012.2014.889911

[12] Tone, K. (2002) A Slacks-Based Measure of Super-Efficiency in Data Envelopment Analysis. European Journal of Operational Research, 143, 32-41. http://dx.doi.org/10.1016/S0377-2217(01)00324-1

[13] Ouenniche, J., Xu, B. and Tone, K. (2014) Relative Performance Evaluation of Competing Crude oil Prices’ Volatility Forecasting Models: A Slacks-Based Super-Efficiency DEA Model. American Journal of Operations Research, 4, 235-245. http://dx.doi.org/10.4236/ajor.2014.44023

[14] Tone, K. (2001) A Slacks-Based Measure of Efficiency in Data Envelopment Analysis. European Journal of Operational Research, 130, 498-509. http://dx.doi.org/10.1016/S0377-2217(99)00407-5

[15] Cooper, W.W., Seiford, L.M. and Tone, K. (2007) Data Envelopment Analysis: A Comprehensive Text with Models, Applications, References and DEA-Solver Software. 2nd Edition, Springer, New York.

[16] Efron, B. (1979) Bootstrap Methods: Another Look at the Jackknife. Annals of Statistics, 7, 1-26. http://dx.doi.org/10.1214/aos/1176344552

[17] Efron B. and Tibshirani R. (1993) An Introduction to the Bootstrap. New York: Chapman \& Hall, CRC Press. http://dx.doi.org/10.1007/978-1-4899-4541-9

[18] Fisher, R.A. (1915) Frequency Distribution of the Values of the Correlation Coefficient in Samples from an Indefinitely Large Population. Biometrika, 10, 507-521. http://dx.doi.org/10.2307/2331838

[19] Xu, B. and Ouenniche, J. (2011) A Multidimensional Framework for Performance Evaluation of Forecasting Models: Context-Dependent DEA. Applied Financial Economics, 21, 1873-1890. http://dx.doi.org/10.1080/09603107.2011.597722

[20] Xu, B. and Ouenniche, J. (2012) A Data Envelopment Analysis-Based Framework for the Relative Performance Evaluation of Competing Crude oil Prices’ Volatility Forecasting Model. Energy Economics, 34, 576-583. http://dx.doi.org/10.1016/j.eneco.2011.12.005

[21] Chang, T.S., Tone, K. and Wu, C.H. (2014) Past-Present-Future Intertemporal DEA Models. Journal of the Operational Research Society, 214, 73-98. 